\title{
Long-Lived Bloch Oscillations with Bosonic Sr Atoms and Application to Gravity Measurement at the Micrometer Scale
}

\author{
G. Ferrari, N. Poli, F. Sorrentino, and G. M. Tino \\ Dipartimento di Fisica and LENS, Università di Firenze, INFN, INFM-CNR, I-50019 Sesto Fiorentino (FI), Italy
}

(Received 21 April 2006; published 9 August 2006)

\begin{abstract}
We report on the observation of Bloch oscillations on the unprecedented time scale of several seconds. The experiment is carried out with ultracold bosonic ${ }^{88} \mathrm{Sr}$ atoms loaded into a vertical optical standing wave. The negligible atom-atom elastic cross section and zero angular momentum in the ground state makes ${ }^{88} \mathrm{Sr}$ an almost ideal Bose gas, insensitive to typical mechanisms of decoherence due to thermalization and external stray fields. The small size of the system enables precision measurements of forces at micrometer scale. This is a challenge in physics for studies of surfaces, Casimir effects, and searches for deviations from Newtonian gravity predicted by theories beyond the standard model.
\end{abstract}

PACS numbers: 03.75. $-\mathrm{b}, 04.80 .-\mathrm{y}, 32.80 .-\mathrm{t}$

Quantum devices based on ultracold atoms show unprecedented features. Atom interferometry is used for precision inertial sensors [1,2], to measure fundamental constants [3-5], and for testing relativity [6].

Here we show that, using laser-cooled strontium atoms in optical lattices, persistent Bloch oscillations are observed for a time $\simeq 10 \mathrm{~s}$, and gravity is determined with ppm sensitivity on a micrometer scale. The small size enables precision measurements of forces at micrometer scale. This is a challenge in physics for studies of surfaces, Casimir effects [7], and searches for deviations from Newtonian gravity predicted by theories beyond the standard model [8,9]. The insensitivity to stray fields and collisions makes $\mathrm{Sr}$ in optical lattices, a candidate also for future clocks [10], a unique sensor for small-scale forces with better performances and reduced complexity compared to proposed schemes using degenerate Bose [11] or Fermi [12] gases. This enables new experiments on gravity at unexplored short distances.

The confinement of ultracold atoms in optical lattices, regular structures created by interfering laser beams where the atoms are trapped by the dipole force, provides clean model systems to study condensed-matter physics problems [13]. In particular, under the influence of a periodic potential and a weak uniform force, the atomic momentum changes periodically across the first Brillouin zone, a phenomenon known as Bloch oscillations [14]. Bloch oscillations were predicted for electrons in a periodic crystal potential in the presence of a static electric field [15] but could not be observed in natural crystals because of the scattering of electrons by the lattice defects. They were directly observed using atoms in an optical lattice [16].

In our experiment, laser-cooled ${ }^{88} \mathrm{Sr}$ atoms are trapped in a one-dimensional vertical optical lattice. The combination of the periodic optical potential and the linear gravitational potential gives rise to Bloch oscillations at a frequency $\nu_{B}$ given by

$$
\nu_{B}=\frac{m g \lambda_{L}}{2 h}
$$

where $m$ is the atomic mass, $g$ is the acceleration of gravity, $\lambda_{L}$ is the wavelength of the light producing the lattice, and $h$ is Planck constant. Since $\lambda_{L}, m$, and $h$ are well known, the force along the lattice axis can be determined by measuring the Bloch frequency $\nu_{B}$. In order to perform a sensitive force measurement, a long time of coherent evolution of the atomic wave function is required. The most common effects limiting the coherence time for ultracold atoms are perturbations due to electromagnetic fields and atom-atom interactions. ${ }^{88} \mathrm{Sr}$ is in this respect a good choice because in the ground state it has zero orbital, spin, and nuclear angular momentum, which makes it virtually insensitive to stray electric and magnetic fields. In addition, ${ }^{88} \mathrm{Sr}$ has remarkably small atom-atom interactions [17]; this has so far prevented the achievement of Bose-Einstein condensation for this atom $[17,18]$ but becomes an important feature in experiments where collisions lead to a loss of coherence limiting the measurement time and the potential sensitivity.

The experimental setup used in this work is schematically shown in Fig. 1. The method used to produce ultracold $\mathrm{Sr}$ atoms was already described in [19]. The experiment starts with trapping and cooling $\sim 5 \times 10^{7}$ ${ }^{88} \mathrm{Sr}$ atoms at $3 \mathrm{mK}$ in a magneto-optical trap (MOT) operating on the ${ }^{1} S_{0^{-}}{ }^{1} P_{1}$ blue resonance line at $461 \mathrm{~nm}$. The temperature is then further reduced by a second cooling stage in a red MOT operating on the ${ }^{1} S_{0^{-}}{ }^{3} P_{1}$ narrow transition at $689 \mathrm{~nm}$, and finally we obtain $\sim 5 \times 10^{5}$ atoms at $400 \mathrm{nK}$. After this preparation phase, which takes about $500 \mathrm{~ms}$, the red MOT is switched off and a onedimensional optical lattice is switched on adiabatically in $50 \mu \mathrm{s}$. The lattice potential is originated by a single-mode frequency-doubled Nd:YVO4 laser $\left(\lambda_{L}=532 \mathrm{~nm}\right)$ delivering up to $350 \mathrm{~mW}$ on the atoms with a beam waist of $200 \mu \mathrm{m}$. The beam is vertically aligned and retroreflected by a mirror producing a standing wave with a period $\lambda_{L} / 2=266 \mathrm{~nm}$. The corresponding photon recoil energy is $E_{R}=h^{2} / 2 m \lambda_{L}^{2}=k_{B} \times 381 \mathrm{nK}$. As expected from band theory [14], the amplitude of the oscillation in mo- 


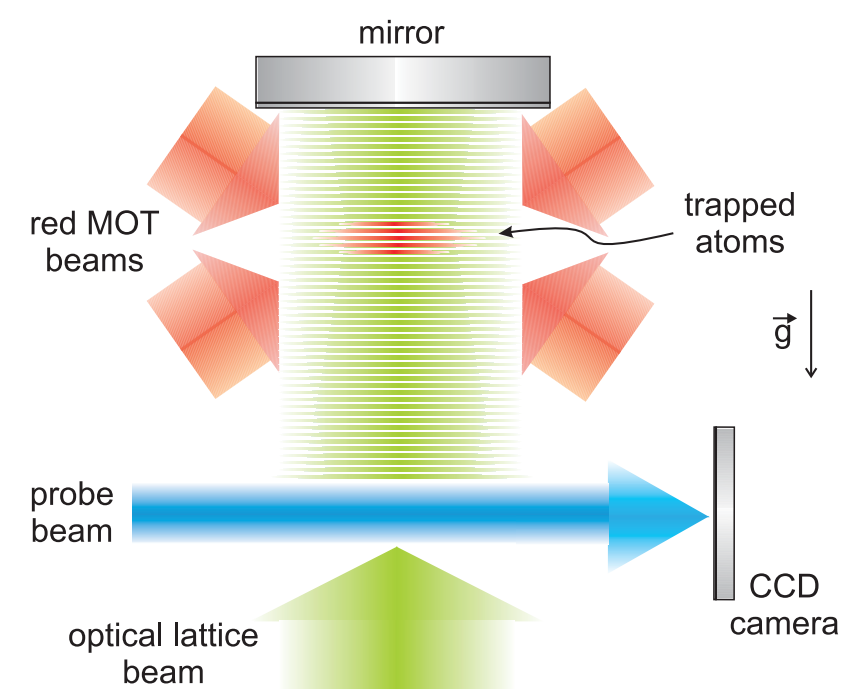

FIG. 1 (color). Simplified scheme of the apparatus used to observe Bloch oscillations and to measure $g$ : Sr atoms are laser cooled and trapped at a temperature of about $400 \mathrm{nK}$ in a red magneto-optical trap (MOT). The MOT laser beams are then switched off and the atoms are transferred in a vertical onedimensional optical lattice generated by a laser beam retroreflected by a mirror; atoms are confined in a series of layers at the antinodes of the standing wave by the dipole force. We measure the momentum distribution of the atoms, after the coherent evolution in the potential given by the periodic potential plus gravity, by a time-of-flight measurement, after a free fall of $12 \mathrm{~ms}$, using a resonant probe laser beam and absorption imaging on a CCD camera.

mentum space decreases as the lattice depth is increased. This suggests that in order to measure the Bloch frequency with maximum contrast the intensity of the lattice laser should be reduced. On the other hand, reducing the intensity results in a loss in the number of trapped atoms because of the smaller radial confinement. For this reason, we used a lattice depth of $10 E_{R}$. For a lattice potential depth corresponding to $10 E_{R}$, the trap frequencies are $50 \mathrm{kHz}$ and $30 \mathrm{~Hz}$ in the longitudinal and radial directions, respectively. For our parameters, the thermal half-width of the momentum distribution is 0.9 times the recoil of the trap photons, thus fulfilling the requirement of a momentum distribution narrower than the first Brillouin zone defined by the lattice potential. Before being transferred in the optical lattice, the atom cloud in the red MOT has a disk shape with a vertical size of $12 \mu \mathrm{m}$ rms. In the transfer, the vertical extension is preserved and we populate about 100 lattice sites with $2 \times 10^{5}$ atoms with an average spatial density of about $10^{11} \mathrm{~cm}^{-3}$. After letting the atoms evolve in the optical lattice, the lattice is switched off adiabatically and the momentum distribution of the sample is measured by a time-of-flight scheme, after a free fall of $12 \mathrm{~ms}$, using a resonant probe laser beam and absorption imaging on a CCD camera.

Figure 2 shows time-of-flight images of the atoms recorded for different times of evolution in the optical lattice potential after switching off the MOT. In the upper part of the frames, the atoms confined in the optical lattice can be seen performing Bloch oscillations due to the combined effect of the periodic and gravitational potential. The average force arising from the photon recoils transferred to the atoms compensates gravity.

The images of trapped atoms obtained by absorption imaging, as the ones shown in Fig. 2, are integrated along the horizontal direction and fitted with the sum of two Gaussian functions. From each image, two quantities are extracted that are reported in Fig. 3 as a function of the evolution time in the lattice: the first [Fig. 3(a)] is the position of each peak in the vertical momentum distribution of the oscillating atoms, showing the expected sawtooth behavior [16]. The second is the width of the atomic momentum distribution (i.e., the second moment of the distribution), which is shown in Fig. 3(b). We find that the latter is less sensitive against noise-induced perturbations to the vertical momentum. We observed $\sim 4000$ Bloch oscillations in a time $t=7 \mathrm{~s}$. During this time, about 8000 photon momenta are coherently transferred to the atoms. Oscillations continue for several seconds, and the measured damping time of the amplitude is $\tau \sim 12 \mathrm{~s}$. To our knowledge, the present results for the number of Bloch oscillations, duration, and the corresponding number of coherently transferred photon momenta are by far the highest ever achieved experimentally in any physical system.

By measuring the initial and final phase of the oscillation on a $7 \mathrm{~s}$ interval, and by counting the number of cycles, we measure the Bloch frequency $\nu_{B}=574.568(3) \mathrm{Hz}$, which corresponds to a gravity acceleration along the optical lattice $g=9.80012(5) \mathrm{ms}^{-2}$. The present sensitivity is

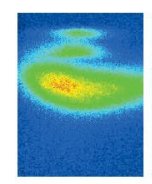

$2.4 \mathrm{~ms}$
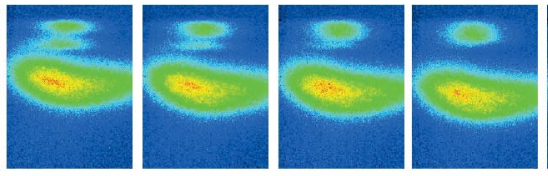

$3.2 \mathrm{~ms}$
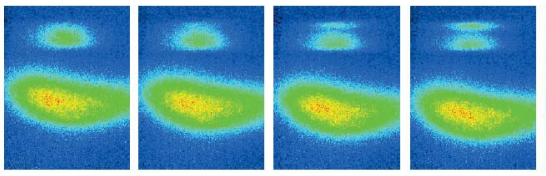

$4.0 \mathrm{~ms}$
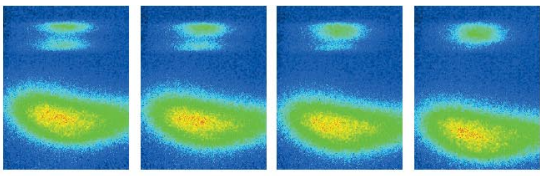

$4.8 \mathrm{~ms}$

FIG. 2 (color). Time-of-flight images of the atoms recorded for different times of evolution in the optical lattice potential after switching off the MOT. In the upper part of each frame, the atoms confined in the optical lattice perform Bloch oscillations for the combined effect of the periodic and gravitational potential. The average force arising from the photon recoils transferred to the atoms compensates gravity. In the lower part, untrapped atoms fall down freely under the effect of gravity. 


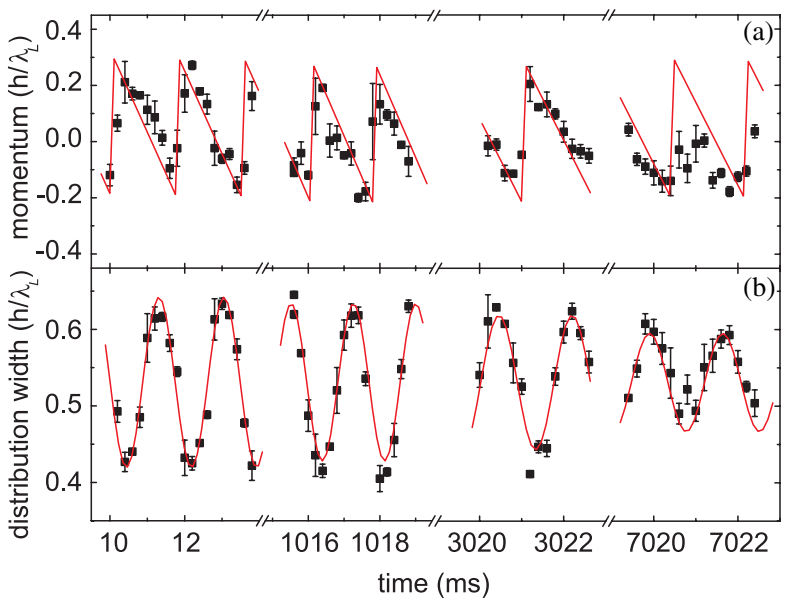

FIG. 3 (color online). Bloch oscillation of ${ }^{88} \mathrm{Sr}$ atoms in the vertical one-dimensional optical lattice under the effect of gravity. Two quantities are extracted from the analysis of the data: (a) the vertical momentum of the oscillating atoms and (b) the width of the atomic momentum distribution. From the fit of the data in (b), a Bloch frequency $\nu_{B}=574.568(3) \mathrm{Hz}$ is obtained with a damping time $\tau \sim 12 \mathrm{~s}$ for the oscillations. Each point is obtained by averaging over four measurements, and the error bars correspond to the mean standard deviation.

$5 \times 10^{-6} \mathrm{~g}$. We expect that a sensitivity of $10^{-7} \mathrm{~g}$ can be achieved using a larger number of atoms and reducing the initial temperature of the sample. Apart from collisional relaxation, which contributes to decoherence on a minute time scale, the main perturbation to quantum evolution is represented by vibrations of the retroreflecting mirror [20]. Negligible contributions to decoherence come from the axial momentum dispersion of the lattice due to its radial size.

Figure 4 illustrates the change of visibility of the twocomponent momentum distribution at the Bragg reflection as a function of the evolution time. The persistent visibility of the bimodal distribution with more than $10^{4}$ atoms reflects the single atom coherent evolution time scale of several seconds, while the reduction in the signal-to-noise ratio is due to the $5 \mathrm{~s}$ lifetime of the sample limited essentially by residual background vapor pressure in the MOT chamber. It is worth noting that the $5 \mathrm{~s}$ lifetime is not inconsistent with the observed $12 \mathrm{~s}$ coherence time: The atoms that collide with the room temperature background vapor are ejected from the $\mu \mathrm{K}$-deep lattice potential, reducing the signal-to-noise ratio but not affecting the amplitude of the oscillation.

Although the sensitivity is lower than the one achievable with other atom interferometry sensors [1], the small spatial extension of the atomic cloud and the possibility to load it into the optical potential at micrometric distance from a surface makes the scheme we demonstrated particularly suitable for the investigation of forces at small spatial scales. The possibility of investigating the gravitational force at small distances by atomic sensors was

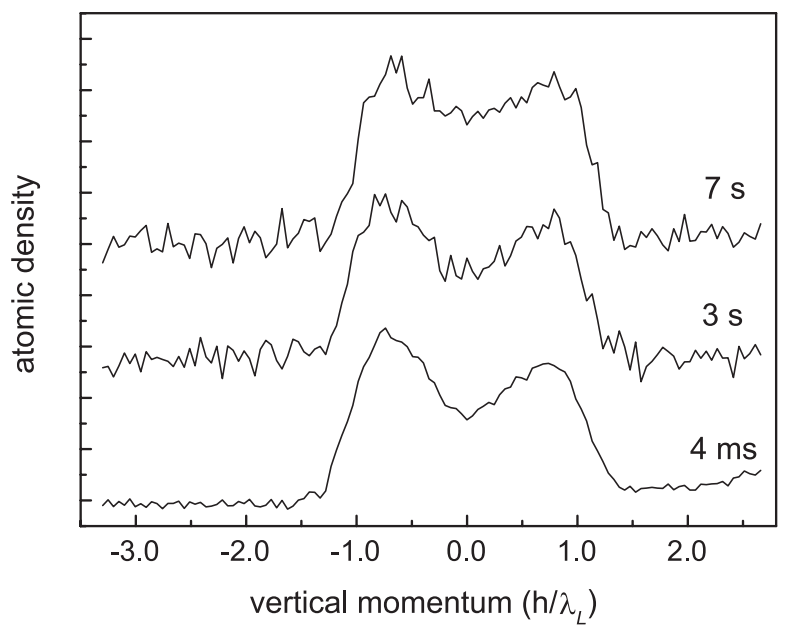

FIG. 4. Vertical momentum distribution of the atoms at the Bragg reflection recorded after an evolution time in the lattice of $4 \mathrm{~ms}, 3 \mathrm{~s}$, and $7 \mathrm{~s}$. Bloch oscillations can be detected with a good contrast for times as long as $7 \mathrm{~s}$ with more than $10^{4}$ atoms. At longer times the signal-to-noise ratio is degraded due to loss of atoms induced by collisions with the background vapor pressure. For a better visibility the vertical scale was rescaled among the three graphs.

proposed in [21], discussed in detail in [22], and preliminarily demonstrated in [23]. Deviations from the Newtonian law are usually described assuming a Yukawa-type potential

$$
V(r)=-G \frac{m_{1} m_{2}}{r}\left(1+\alpha e^{-r / \lambda}\right),
$$

where $G$ is Newton gravitational constant, $m_{1}$ and $m_{2}$ are the masses, and $r$ is the distance between them. The parameter $\alpha$ gives the relative strength of departures from Newtonian gravity and $\lambda$ is its spatial range. Experiments searching for possible deviations have set bounds for the parameters $\alpha$ and $\lambda$. Recent results using microcantilever detectors lead to extrapolated limits $\alpha \sim$ $10^{4}$ for $\lambda \sim 10 \mu \mathrm{m}[8,9]$, while for distances below $10 \mu \mathrm{m}$ it was not possible to perform direct experiments so far. The small size and high sensitivity of the atomic probe allows a direct, model-independent measurement at distances of a few $\mu \mathrm{m}$ from the source mass with no need for modeling and extrapolation as in the case of macroscopic probes. This allows one to directly access unexplored regions in the $\alpha-\lambda$ plane. Also, in this case, quantum objects are used to investigate gravitational interaction.

If we consider a thin layer of a material of density $\rho$ and thickness $d$, the Newtonian gravitational acceleration due to the source mass is $a=2 \pi G \rho d$; for $d \sim 10 \mu \mathrm{m}$ and $\rho \simeq$ $20 \mathrm{~g} / \mathrm{cm}^{3}$, as for gold or tungsten, the resulting acceleration is $a \sim 10^{-10} \mathrm{~ms}^{-2}$. Measuring $\nu_{B}$ at a distance of $10 \mu \mathrm{m}$ from the surface will then provide a direct test of present constraints on $\alpha$ [9]. For smaller distances, around $5 \mu \mathrm{m}$, it is possible to improve present limits on $\alpha$ by more 
than 2 orders of magnitude in the corresponding $\lambda$ range. Even shorter distances could probably be accessed, also considering a related scheme based on a $\mathrm{Sr}$ lattice clock [24]. Nongravitational effects (van der Waals and Casimir forces), also present in other experiments, can be reduced by using a conductive screen and performing differential measurements with different source masses placed behind it. Also, by performing the experiment with different isotopes of Sr, having different masses but the same electronic structure, gravitational forces can be distinguished from other surface interactions.

In conclusion, we observed persistent Bloch oscillations of weak-interacting bosonic $\mathrm{Sr}$ atoms in a vertical optical lattice for a time longer than $7 \mathrm{~s}$, with more than 8000 photon momenta coherently transferred to the atoms. In addition to the intrinsic interest of the observed effect, these results can be important for different experiments. The small size and high sensitivity of the new atomic sensor enable the investigation of small spatial-scale forces as in atom-surface interactions, surface-induced decoherence, Casimir-Polder interaction, and for the search of recently predicted deviations from the Newtonian gravitational law at micrometer scale. Compared to other proposals based on a Bose-Einstein condensate or a degenerate Fermi gas as a probe, the present scheme is not affected by collisional and mean-field degrading effects. In addition, $\mathrm{Sr}$ atoms are insensitive to stray electric and magnetic fields. This leads to longer observation times and higher sensitivity and will allow us to investigate gravity at small spatial scales in unexplored regions.

We thank M. Artoni, G. Oppo, and C. Salomon for a critical reading of the manuscript, G. Saccorotti for the seismic noise measurements, and R. Ballerini, M. De Pas, M. Giuntini, and A. Hajeb for technical assistance. G. M. T. acknowledges stimulating discussions with E. A. Cornell. This work was supported by Istituto Nazionale di Fisica Nucleare (MAGIA experiment), Ente CRF, and EU under Contract No. RII3-CT-2003-506350.

[1] A. Peters, K. Y. Chung, and S. Chu, Nature (London) 400, 849 (1999).

[2] T.L. Gustavson, A. Landragin, and M. A. Kasevich, Classical Quantum Gravity 17, 2385 (2000).
[3] A. Wicht, J. M. Hensley, E. Sarajlic, and S. Chu, Phys. Scr. t102, 82 (2002)

[4] P. Cladé, E. de Mirandes, M. Cadoret, S. Guellati-Khélifa, C. Schwob, F. Nez, L. Julien, and F. Biraben, Phys. Rev. Lett. 96, 033001 (2006).

[5] J. Stuhler, M. Fattori, T. Petelski, and G. M. Tino, J. Opt. B 5, S75 (2003).

[6] S. Fray, C. A. Diez, T. W. Hänsch, and M. Weitz, Phys. Rev. Lett. 93, 240404 (2004).

[7] M. Antezza, L. P. Pitaevskii, and S. Stringari, Phys. Rev. Lett. 95, 113202 (2005).

[8] J. C. Long, H. W. Chan, A. B. Churnside, E. A. Gulbis, M. C. M. Varney, and J.C. Price, Nature (London) 421, 922 (2003).

[9] S. J. Smullin, A. A. Geraci, D. M. Weld, J. Chiaverini, S. Holmes, and A. Kapitulnik, Phys. Rev. D 72, 122001 (2005).

[10] M. Takamoto, F.-L. Hong, R. Higashi, and H. Katori, Nature (London) 435, 321 (2005).

[11] B. P. Anderson and M. A. Kasevich, Science 282, 1686 (1998).

[12] I. Carusotto, L. Pitaevskii, S. Stringari, G. Modugno, and M. Inguscio, Phys. Rev. Lett. 95, 093202 (2005).

[13] I. Bloch, Nature Phys. 1, 23 (2005), and references therein.

[14] N. Ashcroft and N. Mermin, Solid State Physics (Saunders, Philadelphia, 1976).

[15] F. Bloch, Z. Phys. 52, 555 (1929).

[16] M. Raizen, C. Salomon, and Q. Niu, Phys. Today 50, No. 7, 30 (1997), and references therein.

[17] G. Ferrari, R. E. Drullinger, N. Poli, F. Sorrentino, and G. M. Tino, Phys. Rev. A 73, 023408 (2006).

[18] T. Ido, Y. Isoya, and H. Katori, Phys. Rev. A 61, 061403(R) (2000).

[19] N. Poli, R. E. Drullinger, G. Ferrari, J. Léonard, F. Sorrentino, and G. M. Tino, Phys. Rev. A 71, 061403(R) (2005).

[20] Independent measurements with an accelerometer at the level of the retroreflecting mirror indicate a seismic noise consistent with the observed damping time.

[21] G. M. Tino, in Proceedings of the JH Workshop, Firenze, 2001, edited by I. Ciufolini et al. (World Scientific, Singapore, 2003); G. M. Tino, Nucl. Phys. B (Proc. Suppl.) 113, 289 (2002).

[22] S. Dimopoulos and A. A. Geraci, Phys. Rev. D 68, 124021 (2003).

[23] D. M. Harber, J.M. Obrecht, J. M. McGuirk, and E. A. Cornell, Phys. Rev. A 72, 033610 (2005).

[24] P. Lemonde and P. Wolf, Phys. Rev. A 72, 033409 (2005). 